Acta Cryst. (2002). A58 (Supplement), C213

\section{FREEZING TRANSITIONS IN MYOGLOBIN STUDIED BY POWDER DIFFRACTION}

J. Wright

ESRF, BP-220, F-38043 Grenoble Cedex, FRANCE

Obtaining good quality single crystals remains one of the major barriers to the study of protein structure. Cryocooling reduces radiation damage and is widely employed for data collection with synchrotron radiation. However, cryocooling frequently leads a reduction in crystal quality, motivating an in-situ powder diffraction study to understand the processes occurring during the freezing of a protein crystal.

Myoglobin (from horse skeletal muscle) has been studied using the high resolution powder diffraction beamline (BM16) at ESRF and we have observed a pair of first order structural phase transitions at $245 \mathrm{~K}$ and $265 \mathrm{~K}$. The transitions occur above the freezing point of the mother liquor, and involve volume expansions of the order $2-3 \%$. Fast cooling through the transitions traps the sample in the high temperature form, but introduces severe peak broadening, indicating a reduction in the crystal quality. Growth of the $250 \mathrm{~K}$ phase over the course of several hours has been observed at constant temperature, as well as peakshape annealing effects during heating.

Although these transitions might be catastrophic for a single crystal experiment, they can be actively exploited in powder data, since the change in unit cell parameters partially alleviates the peak overlap problem. This leads to a significant increase in the amount of information that can be extracted from a powder diffraction experiment.

\section{Keywords: POWDER DIFFRACTION MYOGLOBIN PHASE} TRANSITIONS
Acta Cryst. (2002). A58 (Supplement), C213

STRUCTURE OF BINARY CaO- $\mathrm{Al}_{2} \mathrm{O}_{3}$ AND SrO- $\mathrm{Al}_{2} \mathrm{O}_{3} \mathrm{LIQUIDS}$ BY COMBINED LEVITATION-NEUTRON DIFFRACTION C. Benmore $^{1}$ J.K.R. Weber ${ }^{2}$ J.A. Tangeman ${ }^{2}$ J. Siewenie $^{1}$ K.J. Heira ${ }^{2}$ ${ }^{1}$ IPNS, Argonne National Laboratory, Argonne, IL 60439, USA ${ }^{2}$ Containerless Research Inc., 906 University Place, IL 60201, USA

The total structure factors, $\mathrm{S}(\mathrm{Q})$ of 50:50 mole $\% \mathrm{CaO}: \mathrm{Al}_{2} \mathrm{O}_{3}{ }^{-}$and 67:33 mole $\% \mathrm{SrO}: \mathrm{Al}_{2} \mathrm{O}_{3}$ composition liquids have been determined by neutron diffraction. Measurements were made on laser beam heated liquids at 2000-2100 K held in an aerodynamic levitator. Pure vanadium levitation nozzles were used. Reduction of the nozzle wall thickness decreased attenuation of scattered neutrons to give a twofold increase in signal intensity. The liquids comprise predominantly 4-coordinate aluminum with the group II metal in 6-and higher coordination with oxygen.

\section{Keywords: LEVITATION NEUTRON DIFFRACTION}

\section{Acta Cryst. (2002). A58 (Supplement), C213}

ON STRUCTURAL PHASE TRANSITIONS IN PEROVSKITES

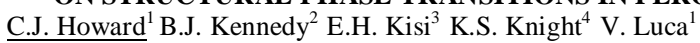

${ }^{1}$ Australian Nuclear Science and Technology Organisation, Private Mail Bag 1, Menai, NSW 2234, Australia ${ }^{2}$ School of Chemistry, University of Sydney, NSW 2006, Australia ${ }^{3}$ Department of Mechanical Engineering, University of Newcastle, NSW 2308, Australia ${ }^{4}$ ISIS Facility, Rutherford Appleton Laboratory, Chilton, Didcot, Oxfordshire OX11 0QX,

The perovskite $\left(\mathrm{ABX}_{3}\right)$ structure comprises a three dimensional corner-linked array of $\mathrm{BX}_{6}$ octahedra with $\mathrm{A}$ cations located between them. The ideal perovskite is cubic, but most perovskites are distorted variants. The most common distortions are cation displacements (identified with irreps $\mathrm{GM}^{4-}, \mathrm{M}^{3-}$ ) and tilting of the $\mathrm{BX}_{6}$ octahedra (irreps $\mathrm{M}^{3+}, \mathrm{R}^{4+}$ ). Though the distortions may be subtle, the transitions between the different structural variants can result in dramatic changes in physical properties, leading to a variety of applications.

The subtlety of the distortions makes for challenging crystallography. With the distortions identified, group theory can be applied [1] to enumerate the possible space groups and structures, and to analyze the transitions between them. Experimental techniques used to elucidate structures include synchrotron-based $\mathrm{X}$-ray diffraction and electron microdiffraction. In studies of the temperatureinduced phase transitions, the combination of group theoretical analysis with very high resolution neutron powder diffraction patterns recorded (using HRPD at ISIS) at fine temperature intervals has proved particularly effective. The sequence of phases in srzro $_{3}$ has been re-examined, and a new high temperature monoclinic phase of perovskite-like $\mathrm{WO}_{3}$ revealed. Results will be presented from recent measurements on the low temperature structures of pralo $_{3}$. All results obtained are consistent with expectations from the group theoretical analysis.

References

[1] Thanks to ISOTROPY, a software package developed by Stokes and Hatch at Brigham Young University, and available at www.physics.byu.edu/stokesh/isotropy.html

\section{Acta Cryst. (2002). A58 (Supplement), C213}

THE EUROPEAN BIOINFORMATICS INSTITUTE MACROMOLECULAR STRUCTURE DATABASE (E-MSD) K. Henrick D Dimitropoulos P Keller J Ionides E Krissinel S Velankar H Boutselakis P McNeil A Golovin J Pineda

EMBL Outstation Hinxton European Bioinformatics Institute Welcome Trust Genome Campus Hinxton Cambridge CB10 1SD UK

Computational protein structure analysis is the basis for understanding protein function. To manage the raw three dimensional structure data contained in the Protein Data Bank (PDB), the EBI Macromolecular Structure Database (EMSD) has utilized the most advanced relational database technology. Strict normalization guidelines and biological as well as chemical consistency checks are enforced to ensure global integrity and quality. Essential components of the E-MSD are a complete chemical description of all the distinct chemical components found within the PDB, the recognition of the oligomeric state of the structure studied, the recognition of secondary structure elements in a fast search method, and the building of cross references from primary sequence. In addition in the transformation of raw data in the relational database aspects of validation, data harvesting and extension of the data base to include additional NMR data and cryo-EM microscopy results have been undertaken.

Keywords: MACROMOLECULAR STRUCTURE RELATIONAL DATABASE BIOINFORMATICSTHE 